Article

\title{
Inverse Design of Artificial Materials Based Lens Antennas through the Scattering Matrix Method
}

\author{
Roberta Palmeri *(1) and Tommaso Isernia \\ Department of Information Engineering, Infrastructure and Sustainable Energy, Università Mediterranea of \\ Reggio Calabria, 89122 Reggio Calabria, Italy; tommaso.isernia@unirc.it \\ * Correspondence: roberta.palmeri@unirc.it; Tel.: +39-0965-169-3285
}

Received: 10 March 2020; Accepted: 23 March 2020; Published: 27 March 2020

check for updates

\begin{abstract}
The design of spatially varying lens antennas based on artificial materials is of high interest for their wide range of applicability. In this paper, we propose a novel design procedure relying on an inverse formulation of the scattering matrix method (SMM). Differently from many adopted approaches, which resort to global optimizations or homogenization procedures, the inverse SMM (I-SMM) allows the synthesis of optimal parameters (geometrical and/or electromagnetic) for the inclusions realizing the overall device in a very effective manner. With reference to the 2D TM case, the proposed tool has been successfully assessed through the synthesis of different kinds of lenses radiating a pencil beam.
\end{abstract}

Keywords: antennas; artificial materials; inverse design; lens antenna; optimization problem; scattering

\section{Introduction}

Lens antennas whose electromagnetic characteristics vary in space deserve a high interest in the literature because of their potential application to different purposes, starting from the enhancement of antenna performance, to imaging or automotive radar applications [1]. In fact, the peculiar feature of lenses is the manipulation of phase or amplitude (or both), as well as of the primary feed radiation in order to modify the latter according to some initial specification.

The Luneburg lens [2] and the (Half) Maxwell Fish Eye lens [3] are the two most famous types of graded dielectric lenses accomplishing useful goals. In fact, the radially varying dielectric permittivity allows the conversion of a plane wave into a spherical wave (Luneburg and Half Maxwell Fish Eye), and vice-versa, while in the case of the Luneburg lens (and the full Maxwell Fish Eye), their circular symmetry allows the radiation properties to obey a rigid steering of the field in the case of an angular variation of the primary source.

On the other hand, at least two issues have to be carefully considered. First, a practical fabrication of such a graded index permittivity distribution is not an easy task. In fact, it usually requires the discretization of the structure with a finite number of shells [4-6], with unavoidable performance deterioration. Second, since the permittivity function is represented by an analytical expression arising from the solution of the Maxwell's equations, both the Luneburg lens and the Maxwell Fish Eye lens can be considered canonical lenses operating in a strictly given fashion, i.e., with assigned and well-defined properties on the radiated patterns.

As far as the fabrication challenge is concerned, in recent years great interest has been devoted to metamaterials-based lenses [7-12]. In fact, by properly adjusting shape, dimension, and arrangement of the constituting sub-wavelength unit cells, it is possible to engineer an effective medium emulating the original permittivity distribution of the lens.

The possibility of changing the lens's response with respect to canonical solutions has been also investigated in the literature in recent years. In this case, the problem becomes how to modify 
the permittivity distribution of a lens in order to generate patterns different from the canonical ones. The transformation optics (TO) theory [13] is the most adopted tool in this contest. In fact, TO aims at tailoring the spatial constitutive profile of a material to properly control the electromagnetic wave propagation. This rigorous formulation has been proved to be effective for a number of applications [14-19], with the relevant drawback being the bi-anisotropy of the arising material.

Recently, a possibility to manage the lens permittivity distribution to achieve desired radiation characteristics has been offered by the inverse scattering problem (ISP) framework [20-23].

It is well known that an ISP [24] is solved if the electromagnetic parameters of an object are determined, in a non-invasive manner, from the knowledge of its scattered field. However, it has been demonstrated in [25] that by substituting the scattered field with assigned field specifications (as long as the specified scattered field has a number of degrees of freedom compatible with the dimensions of the lens), which could be, for example, an enhancement of the field radiated by the Luneburg lens, it is possible to determine the electromagnetic parameters of an object (f.i. the dielectric permittivity of a lens) in such a way to realize the given field (or power pattern) specifications. Recently, a design approach based on the ISP in conjunction with artificial material technology was proposed in [26]. By still adopting the contrast source inversion (CSI) [27] method for the solution of the ISP, in [26] the possibility of enforcing desired properties on the permittivity distribution, as well as of realizing multi-modality lenses, was explored for the first time. Last, but not least, it has been shown that the adoption of a proper representation basis for the unknown, allowing for the direct synthesis of an artificial-materials-based lens, is able to overcome results based on the search of a smoothly varying profile followed by homogenization procedures.

In the same framework, we propose in this contribution a design strategy based on a different tool; that is, the scattering matrix method (SMM) [28]. Introduced by Maystre and co-workers, the SMM allows evaluation in closed form of the field scattered by a set of arbitrarily shaped small inclusions. Basically, it is an analysis tool very efficient from a computational point of view, since it does not require the discretization of the region under test like the method of moments [29], or others.

As a novel contribution, we turn the SMM into a design tool for artificial-materials-based lenses, i.e., we adopt the SMM formulation to retrieve the electromagnetic properties of the constituent small inclusions allowing for the best fitting of the assigned (total) field (or power pattern) specifications.

The remainder of the paper is organized as follows. In Section 2 the SMM is recalled; the inverse SMM (I-SMM) is introduced in Section 3 and assessed in Section 4. Conclusions follow.

\section{The Scattering Matrix Method (SMM)}

Consider the schematic in Figure 1a, wherein a set of scatterers with cross section $C_{i}$ and invariant along the z-direction are depicted in $2 \mathrm{D}$ geometry. Let $\underline{E}_{i n c}$ also be the primary source illuminating the set of scatterers. For simplicity, we suppose a TM polarized incident field, i.e., $\underline{E}_{i n c}=E_{i n c} \hat{i}_{z}$, so that the problem reduces to a scalar one.

We know from the scattering theory that the scattered field obeys the non-homogenous Helmholtz's equation. By considering an expansion in cylindrical harmonics, the overall scattered field can be written as [30]:

$$
E_{s c}(\underline{P})=\sum_{i=1}^{N} E_{s c}^{i}(\underline{P})=\sum_{i=1}^{N} \sum_{m=-\infty}^{+\infty} b_{i, m} H_{m}^{(2)}\left(k_{b} \hat{r}_{i}\right) e^{j m \hat{\theta}_{i}},
$$

where $\left(\hat{r}_{i}, \hat{\theta}_{i}\right)$ represents the coordinates of a generic observation point with respect to the $i$-th scatterer, $k_{b}$ denotes the wavenumber of the background medium, $H_{m}^{(2)}(\cdot)$ is the second kind $m$-th order Hankel function, and $N$ is the total number of scatterers.

Equation (1) represents the scattered field as the sum of the fields scattered from each cylinder, where each of these contributions is expressed in a reference system centered in the $i$-th scatterer. By applying Graf's formula for Hankel functions [31], it is possible to express the field scattered by the 
$i$-th target in a single coordinates system. For example, if we use the reference system centered in the $\ell$-th scatterer, $C_{\ell}$, one has [28]:

$$
E_{S C}^{i}(\underline{P})=\sum_{m=-\infty}^{+\infty} b_{i, m} \sum_{q=-\infty}^{+\infty} e^{j(m-q) \theta_{\ell}^{i}} H_{q-m}^{(2)}\left(k_{b} r_{\ell}^{i}\right) J_{q}\left(k_{b} \hat{r}_{\ell}\right) e^{j q \hat{\theta}_{\ell}},
$$

which holds if $\hat{r}_{\ell} \leq r_{\ell}^{i}$. In Equation (2), $r_{\ell}^{i}$ and $\theta_{\ell}^{i}$ are the relative distance and angle between the two systems of coordinates, respectively, while $\left(\hat{r}_{\ell}, \hat{\theta}_{\ell}\right)$ represents the coordinates of the observation point with respect to the $\ell$-th scatterer (or reference system); see Figure 1 b,c.

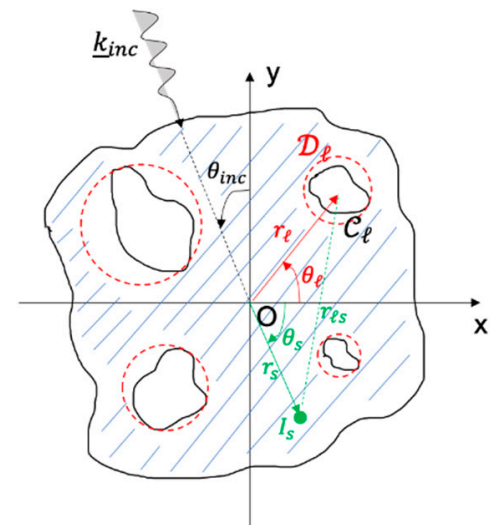

(a)

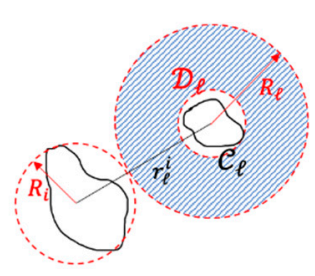

(b)

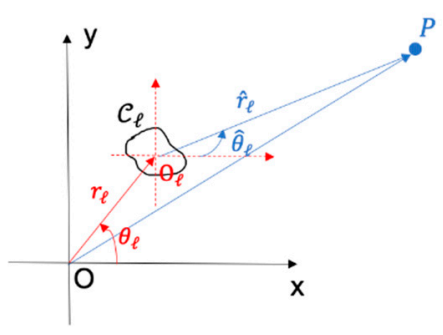

(c)

Figure 1. Geometrical sketch and notation involved in the scattering matrix method (SMM). In (a) both the cases of plane wave and line current incident fields are depicted. In (b) an enlargement of two generic scatterers is depicted to define reciprocal variables and highlight the annulus wherein the scattered field can be evaluated through the modal expansion. In (c) the coordinates of the observation point are defined with respect to the generic $\ell$-th reference system.

Finally, by considering the representation of the incident field in the chosen reference system, the total field in the annulus between $\mathcal{D}_{\ell}$ (that is the minimum circle enclosing the scatterer) and the circle with radius $R_{\ell}$ passing through the closest point of the nearest $i$-th cylinder (see Figure $1 \mathrm{~b}$ ) can be evaluated as:

$$
\begin{aligned}
E_{t o t}(\underline{P}) & =E_{i n c}(\underline{P})+\sum_{i=1, i \neq \ell}^{N} E_{s c}^{i}(\underline{P})+\sum_{m=-\infty}^{+\infty} b_{\ell, m} H_{m}^{(2)}\left(k_{b} \hat{r}_{\ell}\right) e^{j m \hat{\theta}_{\ell}} \\
& =\sum_{m=-\infty}^{+\infty} a_{\ell, m} J_{m}\left(k_{b} \hat{r}_{\ell}\right) e^{j m \hat{\theta}_{\ell}}+\sum_{m=-\infty}^{+\infty} b_{\ell, m} H_{m}^{(2)}\left(k_{b} \hat{r}_{\ell}\right) e^{j m \hat{\theta}_{\ell}},
\end{aligned}
$$

where:

$$
\begin{aligned}
& E_{\text {inc }}(\underline{P})=\left\{\begin{array}{cl}
e^{-j k r^{\ell} \sin \left(\theta_{i n c}-\theta^{\ell}\right)} \sum_{m=-\infty}^{+\infty} J_{m}\left(k_{b} \hat{r}_{\ell}\right) e^{j m\left(\hat{\theta}_{\ell}-\theta_{\text {inc }}\right)} & \text { for plane wave incident field } \\
-\frac{\omega \mu_{0} I_{s}}{4} \sum_{m=-\infty}^{+\infty} H_{m}^{(2)}\left(k_{b} r_{\ell s}\right) J_{m}\left(k_{b} \hat{r}_{\ell}\right) e^{j m\left(\hat{\theta}_{\ell}-\theta_{\ell s}\right)} & \text { for line current incident field }
\end{array}\right. \\
& a_{\ell, m}=Q_{\ell, m}+\sum_{i=1, i \neq \ell}^{N} \mathcal{T}_{\ell, i, m, q} b_{i, m} .
\end{aligned}
$$

and (by swapping $q$ and $m$ ) [28]:

$$
\mathcal{T}_{\ell, i, m, q}=e^{j(q-m) \theta_{\ell}^{i}} H_{m-q}^{(2)}\left(k_{b} r_{\ell}^{i}\right),
$$




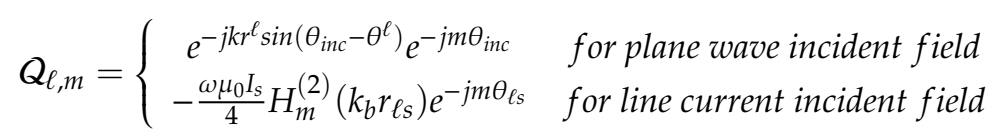

where $\theta_{\text {inc }}$ is the incidence angle with respect to the y-axis, while $I_{s}$ indicates the intensity of the line current and $\left(r_{\ell_{s}}, \theta_{\ell s}\right)$ its coordinates with respect to the $\ell$-th reference system (see Figure 1a).

It is worth noting that the two series in the last row of Equation (3) have a different physical meaning. As a matter of fact, while the second term is simply the field scattered by the $\ell$-th target, the first series represents the 'local' incident field on the $\ell$-th target, that is, the sum of the primary incident field $E_{i n c}$ and the field scattered from all other targets (which acts as a secondary incident field) in the $\ell$-th region at hand.

Equation $(4 \mathrm{~b})$ can be turned into a matrix relation for any scatterer $\mathcal{C}_{\ell}$ as:

$$
\mathbf{a}_{\ell}=\mathbf{Q}_{\ell}+\sum_{i=1, i \neq \ell}^{N} \mathbf{T}_{\ell, i} \mathbf{b}_{i}
$$

where $\mathbf{a}_{\ell}, \mathbf{b}_{i}$, and $\mathbf{Q}_{\ell}$ are the column matrices with elements $a_{\ell, m}, b_{i, m}$, and $Q_{\ell, m}$, respectively, while $\mathbf{T}_{\ell, i}$ is a square blocks matrix of the $(m, q)$-th element $\mathcal{T}_{\ell, i, m, q}$.

Moreover, $\mathbf{b}_{\ell}=\mathbf{S}_{\ell} \mathbf{a}_{\ell}$, that is, the so-called scattering matrix $\mathbf{S}_{\ell}$, relates the coefficients of the scattered and incident fields. Hence, by multiplying Equation (5) by $\mathbf{S}_{\ell}$, we finally obtain a linear system in the unknown scattering coefficients $\mathbf{b}_{\ell}$ [28]:

$$
\mathbf{b}_{\ell}-\sum_{i=1, i \neq \ell}^{N} \mathbf{S}_{\ell} \mathbf{T}_{\ell, i} \mathbf{b}_{i}=\mathbf{S}_{\ell} \mathbf{Q}_{\ell} .
$$

Once Equation (6) is solved, the scattered field in the region of space outside scatterers can be finally evaluated through Equation (1).

Some comments highlighting the advantages of using the SMM are now in order. As long as we are dealing with an analysis problem, the electromagnetic parameters of each scatterer, encoded in the scattering matrix $\mathbf{S}_{\ell}$, are known. Hence, the individual $\mathbf{S}_{\ell}$ matrix can be evaluated independently from the main code once and for all. Moreover, if all the scatterers are identical, matrices $\mathbf{S}_{\ell}$ will also be identical because they are defined in the local coordinate system centered in each cylinder. Finally, in case of scatterers with circular symmetry (which is the case considered in this paper), each $\mathbf{S}_{\ell}$ reduces to a diagonal matrix whose elements can be expressed in closed form.

\section{Inverse Design Through the Scattering Matric Method (I-SMM)}

Solving an inverse design problem concerns the retrieval of geometrical, electromagnetic, and/or other parameters of an object. In this respect, the adoption of the SMM as a design tool implies that, besides the scattering coefficients $\mathbf{b}_{\ell}$, the scattering matrices $\mathbf{S}_{\ell}$ are also unknown. Consequently, Equation (6) turns into a system of non-linear equations, like the usual ISP. However, since we are not dealing with a diagnosis problem, the so called 'false solutions' [32] related to the non-linearity of the problem are no longer an issue. In fact, whatever solution is able to satisfy the assigned specifications is an admissible solution. This means that one can achieve several solutions obeying the same specifications. Interestingly, through the I-SMM we are also able to handle different kinds of scatterers by just considering the pertinent formulation for the scattering matrices entries $s_{m}$.

In this paper, we focus on cylindrical scatterers with circular symmetry, so that $\mathbf{S}_{\ell}$ are diagonal matrices. Then, by changing the expression for $s_{m}$, we can deal with dielectric, magnetic, metallic, stratified, or other kinds of scatterers. For the sake of completeness, analytic expressions for a number of cases of interest are reported in Appendix A.

Let $\widetilde{E}_{t o t}(\underline{R})$ be the assigned specification for the total field on a surface identified by $\underline{R}$. For the sake of generality, also consider $\xi$ to be the vector containing the unknowns relative to inclusion 
properties; in more detail, entries of $\xi$ can be dielectric permittivity, magnetic permeability, radii, or any combination of these. The unknown $\tau$ is related instead to the complex amplitude of the (fixed pattern) primary source. Therefore, the design problem can be formulated as it follows:

$$
\min _{\xi, \tau} \frac{\left\|\widetilde{E}_{t o t}(\underline{R})-E_{\text {tot }}^{o p t}(\underline{R})\right\|_{2}^{2}}{\left\|\widetilde{E}_{t o t}(\underline{R})\right\|_{2}^{2}} \text { subject to physical constraints on } \xi,
$$

where:

$$
\begin{gathered}
E_{\text {tot }}^{\text {opt }}(\underline{R})=\tau E_{\text {inc }}(\underline{R})+E_{\text {scat }}^{\text {opt }}(\underline{R}), \\
E_{\text {scat }}^{o p t}(\underline{R})=\sum_{i=1}^{N} \sum_{m=-\infty}^{+\infty} b_{i, m}^{o p t} H_{m}^{(2)}\left(k_{b} \hat{r}_{i}\right) e^{j m \hat{\theta}_{i}}, \\
\mathbf{b}_{\ell}^{\text {opt }}-\sum_{i=1, i \neq \ell}^{N} \mathbf{S}_{\ell}^{o p t}(\xi) \mathbf{T}_{\ell, i} \mathbf{b}_{i}^{o p t}=\mathbf{S}_{\ell}^{o p t}(\xi) \mathbf{Q}_{\ell} .
\end{gathered}
$$

In more detail, the optimization problem of Equation (7a) based on the I-SMM aims at retrieving optimal values for $\xi$ and $\tau$ such to minimize the mismatch between the assigned field and the optimal field, while fitting the model of the SMM through the conditions of Equations $(7 \mathrm{~b})-(7 \mathrm{~d})$. Note the complex amplitude $\tau$ of the primary source(s) has been inserted within the unknowns of the CSI functional, as such an additional degree of freedom allows an easier fitting of specifications (see [22,33] for more details).

As far as the unknown vector $\xi$ is concerned, note that whatever the quantities one is looking for (permittivities, radii, or permeabilities), they implicitly define the entries $s_{m}$ of the matrices $\mathbf{S}_{\ell}$, (see Appendix A). Hence, physical constraints on $\xi$ have to guarantee positive values for radii and feasible values for permittivity and permeability.

It is worth highlighting that a number of advantages are in order in using I-SMM rather than the usual inverse scattering formulation [26] for the design of artificial-materials-based devices. First, by virtue of the embedded SMM, the I-SMM is more efficient with respect to [26] from a computational point of view. In fact, in [26] we were forced into a very fine discretization of the region of interest in order to accurately represent the small circular inclusions at hand, while in this case the computational burden just depends on the number of cylinders and on their diameters. Secondly, by exploiting the scattering matrices of the scatterers in the present approach, differently from [26] where just e.m. parameters were looked for, it is possible to look for either electromagnetic or geometrical parameters (radii), or even both.

\section{Design of a Lens Radiating a Pencil Beam: Numerical Assessment}

The proposed design approach has been tested through the synthesis of a circular lens antenna radiating a pencil beam. The lens is supposed to be composed of small (with respect to the operating wavelength) inclusions arranged on eight concentric rings plus the central one. Its overall dimension is $3 \lambda$ with $N=217$ inclusions in total. We refer to this kind of structure as a graded artificial material (GAM) based lens. The primary incident field is a $z$-invariant source having a pattern of the kind $E_{\text {inc }}(\underline{r})=-\frac{\omega \mu_{0} I_{s}}{4} H_{0}^{(2)}\left(k_{b} r\right) \cos ^{2} \vartheta$, with $I_{s}=0.001$ and $\underline{r}=(r, \vartheta)$ being the coordinate of a generic point in the reference system centered on the source, located $0.4 \lambda$ far from the lens's rim.

The aim of the design is to find optimal electromagnetic and/or geometrical parameters for the lens such that its radiated field matches a given field $\widetilde{E}_{t o t}(\underline{R})$ in a certain region of space. As far as the definition of $\widetilde{E}_{t o t}(\underline{R})$ is concerned, it has been done by first solving an optimal synthesis problem as in [34] (and using a properly truncated cylindrical harmonics expansion for the overall scattered field [35]) and then performing a back-propagation of the so-obtained far field pattern to a circumference located in the near-field of the lens [26]. To avoid effects due to the primary feed, the fitting is pursued on an arc of about $215^{\circ}$ in front of the lens. The GAM-based lens is instead supposed to be circularly 
symmetric, i.e., the parameters looked for are supposed to be identical for all the inclusions of the same ring; consequently, there is a number of unknown matrices $\mathbf{S}_{\ell}$ equal to 9.

Finally, before applying the I-SMM, the series involved in the mathematical formulation have to be conveniently truncated. Hence, because of the small dimensions of cylinders, just three harmonics are considered to represent the scattered field (i.e., $m=-1,0,1 ; q=-1,0,1)$. The optimization problem of Equations (7a)-(7d) was solved through the fmincon routine of MATLAB.

To demonstrate the flexibility of the I-SMM approach, we dealt with the design of different types of GAM-based lens by considering different possibilities for the unknown vector $\xi$ in Equations (7a)-(7d). In particular, by following the same terminology as in [26], we can:

- fix the radius $\rho$ for all cylinders and suppose the entries of $\xi$ as the permittivity of inclusions for each ring, i.e., $\varepsilon_{r}^{\ell}$, and hence carry out the design of a $G A M_{R}$-based lens (GAM-based lens with a gradient of the refractive index in the radial direction);

- fix the permittivity $\varepsilon_{r}$ for all cylinders and suppose the entries of $\xi$ as the radius of inclusions for each ring, i.e., $\rho^{\ell}$, and hence carry out the design of a $G A M_{F}$-based lens (GAM-based lens with a gradient of the filling factor);

- $\quad$ consider $\xi=\left[\varepsilon_{r}^{\ell} ; \rho\right]$, that is, search for the radius of all inclusions and their permittivity for each ring at the same time. This kind of structure is still a $G A M_{R}$-based lens;

- $\quad$ opposite to the previous case, consider $\xi=\left[\rho^{\ell} ; \varepsilon_{r}\right]$, i.e., search for the unique permittivity value for all inclusions and their radius for each ring contemporarily. We still achieve a $G A M_{F}$-based lens.

Then, a fifth case where the inclusions are cylindrically stratified (two layers) is also considered. In all cases, specifications on the total far field have been chosen in such a way to maximize the separation amongst the maximum field (in the target direction) and the peak sidelobe (for a given beamwidth).

Results for the above cases are separately reported in the following subsections. For the first two cases, comparisons with results achieved by means of the approach in [26] are carried out; this can be quantitatively appraised in Table 1 in terms of beamwidth (BW) and side lobes level (SLL) of the radiated far field. For the other instances, a comparison cannot be performed since the CSI-based tool does not allow a straightforward design of inclusions' dimensions.

Table 1. Quantitative comparison of performance of different kinds of synthesized lenses.

\begin{tabular}{lcccccc}
\hline & \multicolumn{3}{c}{ BW [deg] } & \multicolumn{3}{c}{ SLL [dB] } \\
\cline { 2 - 7 } & Requirements & CSI & I-SMM & Requirements & CSI & I-SMM \\
\hline Device Sect. 4.1 & 40.32 & 37.5 & 41.8 & -25 & -17.62 & -23.45 \\
Device Sect. 4.2 & 40.32 & $39(@-20 \mathrm{~dB})$ & 38.8 & -25 & -17.41 & -19.15 \\
Device Sect. 4.3 & 40.32 & - & 43.2 & -25 & - & -24.29 \\
Device Sect. 4.4 & 40.32 & - & 40.3 & -25 & - & -19.33 \\
Device Sect. 4.5 & 40.32 & - & 41.7 & -25 & - & -24.72 \\
\hline
\end{tabular}

\section{1. $G A M_{R}$-Based Lens with Fixed Radius}

Consider lossless non-magnetic cylinders with radius $\rho=\lambda / 12$ and fixed arrangement. The synthesized permittivity of the lens is depicted in Figure 2a and was achieved by considering as a starting point of the I-SMM optimization problem the permittivity distribution of the Luneburg lens as discretized on the set of inclusions. By solving the direct problem with the synthesized lens and the obtained value of $\tau$, the radiated far field can be finally computed. It is shown with a green curve in Figure $2 b$ from which is possible to appraise the fulfillment of constraints on SLL (SLL $\leq-25 d B$ ). A comparison with the CSI-based design tool [26] demonstrates the capability of the I-SMM to better handle GAM-based devices, as also evidenced by Table 1 . 


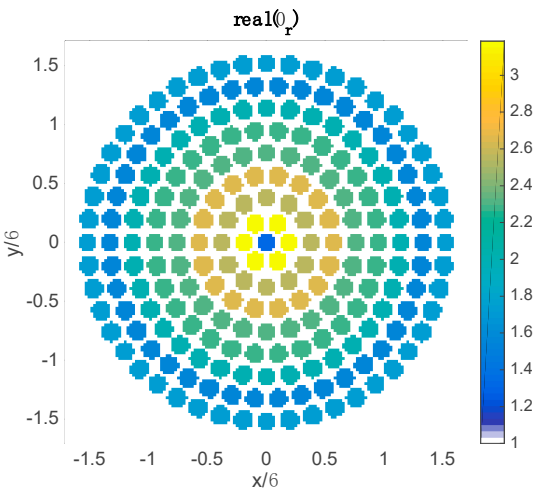

(a)

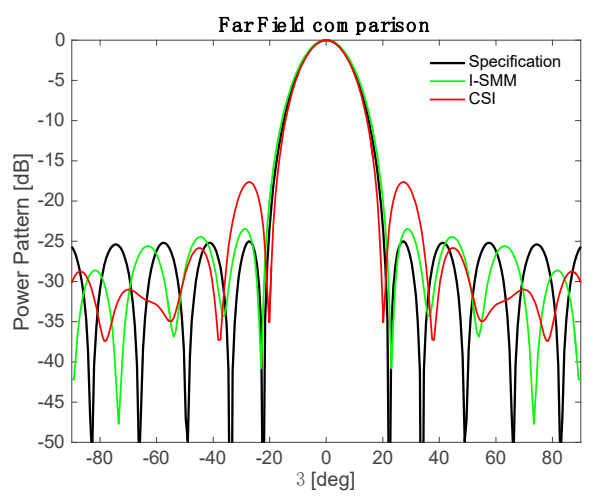

(b)

Figure 2. Synthesis of permittivity values for a $\mathrm{GAM}_{\mathrm{R}}$-based lens with fixed radius $[\tau=-1.3697+$ j0.4214]. (a) Real part of the permittivity function; (b) comparison on radiated far field.

\section{2. $\mathrm{GAM}_{\mathrm{F}}$-Based Lens with Fixed Permittivity}

Consider lossless non-magnetic cylinders whose permittivity is $\varepsilon_{r}=4.5$ and with fixed arrangement. The lens with the synthesized radii is depicted in Figure 3a and was achieved by considering as a starting point of the I-SMM optimization problem cylinders with the same radius $\lambda / 12$.

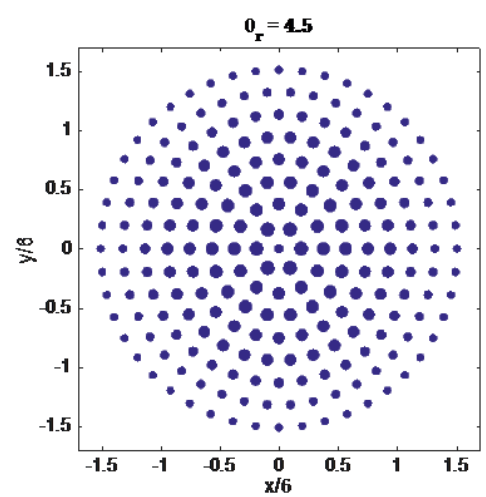

(a)

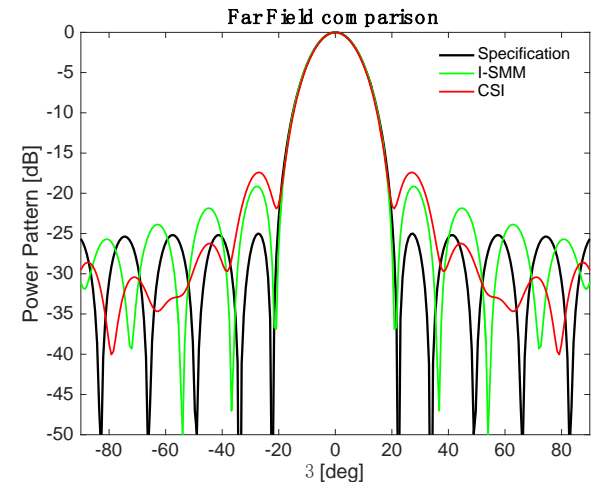

(b)

Figure 3. Synthesis of radius values for a $\mathrm{GAM}_{\mathrm{F}}$-based lens with fixed permittivity [ $\tau=-1.1991-j 0.4658$ ]. (a) Real part of the permittivity function; (b) comparison on radiated far field.

As can be seen from curves in Figure 3b, the performance of the novel I-SMM method and the CSI-based method are comparable in this case (see Table 1). However, it is worth noting that the CSI-based method does not allow the direct synthesis of radii, but the intermediate step of $\mathrm{GAM}_{R}$ design is required to obtain an equivalent $\mathrm{GAM}_{\mathrm{F}}$ device [26].

\section{3. $G A M_{R}$-Based Lens with Unknown (Common) Radius}

In this case the problem is analogous to the one reported in Section 4.1, but with the difference that the radius $\rho$ of the lossless non-magnetic cylinders is also unknown. The achieved value from the synthesis procedure is $\rho=0.0867 \lambda$, which is only slightly different from the one considered in Section 4.1, but which also allows the further reduction of the SLL; see Figure $4 \mathrm{~b}$ and Table 1. 


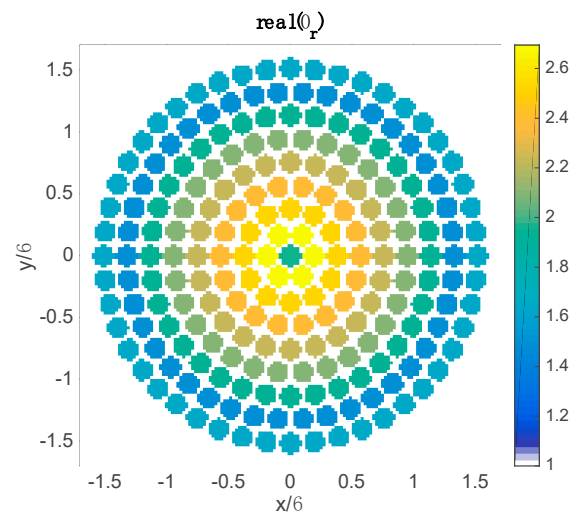

(a)

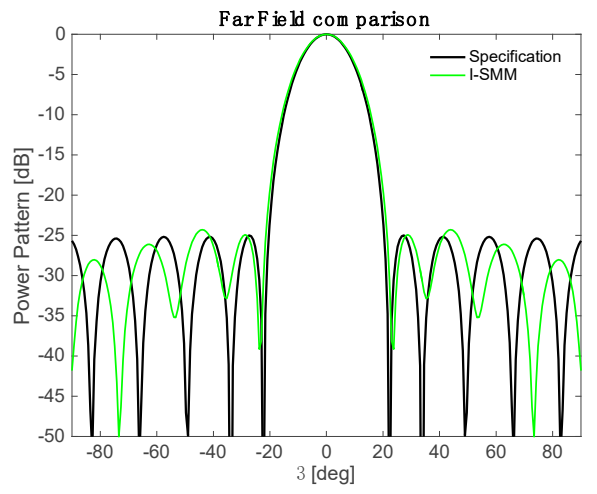

(b)

Figure 4. Synthesis of permittivity values for a $G_{A M}$-based lens with unknown radius $[\tau=-1.2995+j 0.6573]$. (a) Real part of the permittivity function; (b) radiated far field.

\section{4. $G A M_{F}$-Based Lens with Unknown(Common) Permittivity}

The same circumstances of Section 4.2 are considered in the present section, but with also considering unknown the constant permittivity value for all the inclusions. The synthesized value is $\varepsilon_{r}=3.3591$ in this case, allowing a slight reduction of SLL with respect to Section 4.2; see Figure $5 \mathrm{~b}$ and Table 1.

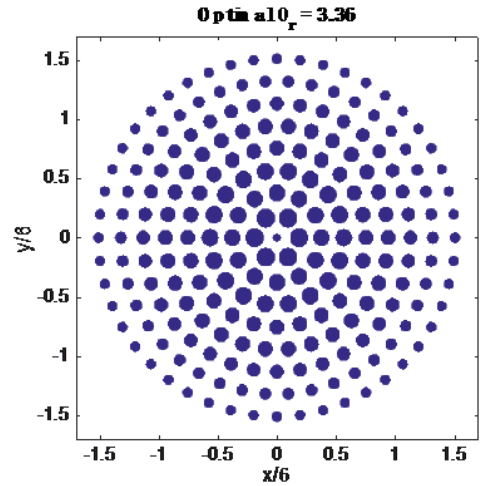

(a)

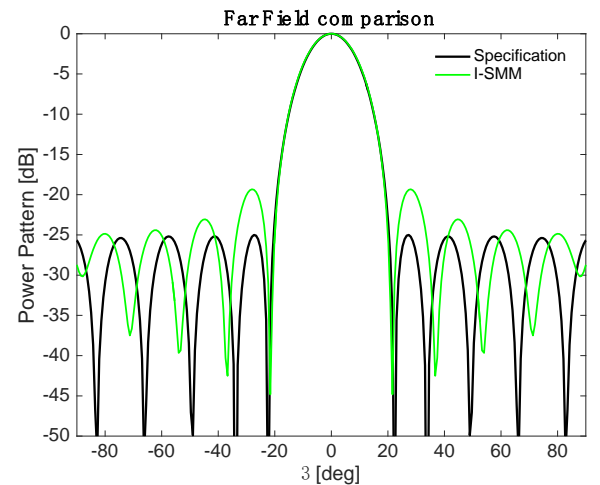

(b)

Figure 5. Synthesis of radius values for a $\mathrm{GAM}_{\mathrm{F}}$-based lens with unknown permittivity $[\tau=-0.9369-j 0.8722]$. (a) Real part of the permittivity function; (b) comparison on radiated far field.

\subsection{Stratified GAM-Based Lens with Fixed Radii}

The last example concerns the design of a GAM-based lens composed of layered inclusions. By considering lossless and non-magnetic cylinders composed of two layers and with fixed positions, two unknowns for each ring have to be synthesized in this case, i.e., the permittivity of the inner and outer layer, while their radii are supposed to be $\rho_{\text {in }}=\lambda / 24$ and $\rho_{\text {in }}=\lambda / 12$, respectively. The synthesized lens is shown in Figure 6a and the corresponding radiated field is depicted with a green curve in Figure $6 \mathrm{~b}$. A quantitative comparison can be appraised from Table 1. As can be observed, very good fulfillment of specifications is achieved with the designed lens. Although the CSI-based tool could deal with the present design problem, a comparison with that is not reported because of the non-reasonable time required for the synthesis in this case, due to the extremely fine discretization required for representing the smaller layer. 


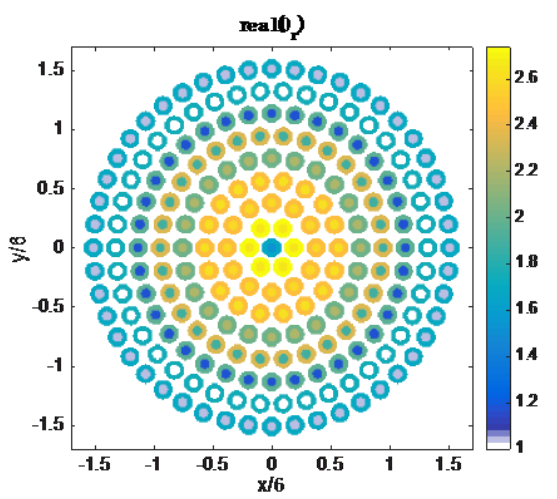

(a)

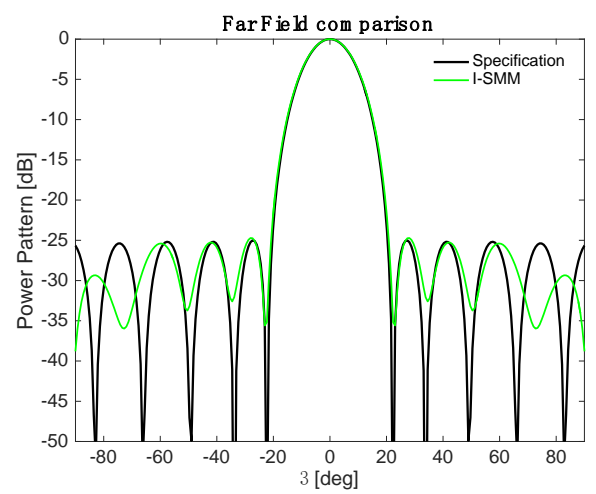

(b)

Figure 6. Synthesis of permittivity values for a GAM-based lens composed of layered inclusions $[\tau=$ $-0.8116+$ j1.3241]. (a) Real part of the permittivity function; (b) Radiated far field.

\section{Conclusions}

In this paper, a design tool for artificial-materials-based lens antennas has been proposed. In particular, the well-known scattering matrix method (SMM), usually adopted for the analysis of structures composed of a set of inclusions, has been conveniently turned into a design tool for the synthesis of inclusions. The proposed inverse SMM (I-SMM) is very flexible in handling different types of artificial-materials-based devices (such as lenses exhibiting gradient in the refractive index or in the filling factor) thanks to the exploitation of the scattering matrices relating the coefficients of incident and scattered fields for each inclusion. Stratified inclusions can be also considered, and possible applications are not limited to antennas. In fact, encouraged by the present outcomes, work is in progress for the optimization of EBG (2D) waveguides and components.

Besides the formulation of the SMM for synthesis is a novelty per se, the proposed approach has shown advantages in terms of both performance and computational burden with respect to the literature, because of its intrinsic nature of naturally dealing with a set of inclusions that is the basic structure of artificial materials. For all the above reasons, the I-SMM is viable in designing metamaterials-based or photonic-crystals-based devices when a 2D scalar problem and an in-plane propagation can be applied. An extension to the out-of-plane propagation and full 3D structures, which would of course further extend the range of possible applications (including the very popular invisibility problem), is deferred to future works.

Author Contributions: Conceptualization, T.I.; validation and investigation, R.P. All authors have read and agreed to the published version of the manuscript.

Funding: This research was funded by project 2017HZJXSZ_003 CYBER-PHYSICAL ELECTROMAGNETIC VISION: Context-Aware Electromagnetic Sensing and Smart Reaction.

Conflicts of Interest: The authors declare no conflict of interest.

\section{Appendix A}

This appendix is devoted to report the analytical formulation of the scattering coefficient $s_{m}$, i.e., the entries of the scattering matrix $\mathbf{S}_{\ell}$, in case of circularly symmetric cylindrical scatterers with radius $\rho$. Note that, under such hypotheses, $\mathbf{S}_{\ell}$ is a diagonal matrix.

To derive the following expressions, harmonic expansions must be written for the electric and magnetic scattered fields in each region (e.g., inside and outside the cylinder) and continuity conditions then applied for the tangential components of both the electric and magnetic fields.

In the following formulas, the subscript " 1 " refers to the outer medium (usually air) and " 2 " to the scatterer. In case of stratified inclusions with two layers, we consider the additional subscript " 3 " to refer to the inner layer of the scatterer; radii will be denoted $\rho_{\text {in }}$ and $\rho_{\text {out }}$. Finally, $J_{n}(\cdot)$ and $H_{n}^{(2)}(\cdot)$ are the $n$-th order Bessel function and Hankel function of the second kind, respectively, while the apex on 
them stands for the first derivative operation. In case of non-magnetic cylinders and air outer medium, $\mu_{3}=\mu_{2}=\mu_{1}=1$.

Analytical form of the scattering coefficients for a metallic cylinder [30]

$$
s_{m}=-\frac{J_{n}\left(k_{1} \rho\right)}{H_{n}^{(2)}\left(k_{1} \rho\right)}
$$

Analytical form of the scattering coefficients for a dielectric cylinder [30]

$$
s_{m}=\frac{\frac{\mu_{1}}{\mu_{2}} k_{2} J_{m}^{\prime}\left(k_{2} \rho\right) J_{m}\left(k_{1} \rho\right)-k_{1} J_{m}\left(k_{2} \rho\right) J_{m}^{\prime}\left(k_{1} \rho\right)}{k_{1} J_{m}\left(k_{2} \rho\right) H_{m}^{(2)^{\prime}}\left(k_{1} \rho\right)-\frac{\mu_{1}}{\mu_{2}} k_{2} J_{m}^{\prime}\left(k_{2} \rho\right) H_{m}^{(2)}\left(k_{1} \rho\right)}
$$

Analytical form of the scattering coefficients for a dielectric cylinder with a dielectric (non-magnetic) cover [36]

$$
\begin{aligned}
& s_{m}=\frac{\alpha \beta+\gamma \delta}{\alpha \varepsilon+\gamma \zeta} \\
& \alpha=\left[-\frac{k_{3}}{\mu_{3}} H_{m}^{(2)}\left(k_{2} \rho_{i n}\right) J_{m}^{\prime}\left(k_{3} \rho_{i n}\right)+\frac{k_{2}}{\mu_{2}} H_{m}^{(2) \prime}\left(k_{2} \rho_{\text {in }}\right) J_{m}\left(k_{3} \rho_{\text {in }}\right)\right] \\
& \beta=\left[\frac{k_{2}}{\mu_{2}} J_{m}\left(k_{1} \rho_{\text {out }}\right) H_{m}^{(1) \prime}\left(k_{2} \rho_{\text {out }}\right)-\frac{k_{1}}{\mu_{1}} J_{m}^{\prime}\left(k_{1} \rho_{\text {out }}\right) H_{m}^{(1)}\left(k_{2} \rho_{\text {out }}\right)\right] \\
& \gamma=\left[-\frac{k_{3}}{\mu_{3}} H_{m}^{(1)}\left(k_{2} \rho_{i n}\right) J_{m}^{\prime}\left(k_{3} \rho_{i n}\right)+\frac{k_{2}}{\mu_{2}} H_{m}^{(1) \prime}\left(k_{2} \rho_{i n}\right) J_{m}\left(k_{3} \rho_{i n}\right)\right] \\
& \delta=\left[\frac{k_{1}}{\mu_{1}} H_{m}^{(2)}\left(k_{2} \rho_{\text {out }}\right) J_{m}^{\prime}\left(k_{1} \rho_{\text {out }}\right)-\frac{k_{2}}{\mu_{2}} H_{m}^{(2) \prime}\left(k_{2} \rho_{\text {out }}\right) J_{m}\left(k_{1} \rho_{\text {out }}\right)\right] \\
& \varepsilon=\left[-\frac{k_{2}}{\mu_{2}} H_{m}^{(2)}\left(k_{1} \rho_{\text {out }}\right) H_{m}^{(1) \prime}\left(k_{2} \rho_{\text {out }}\right)+\frac{k_{1}}{\mu_{1}} H_{m}^{(2) \prime}\left(k_{1} \rho_{\text {out }}\right) H_{m}^{(1)}\left(k_{2} \rho_{\text {out }}\right)\right. \\
& \left.\zeta=\left[-\frac{k_{1}}{\mu_{1}} H_{m}^{(2)}\left(k_{2} \rho_{\text {out }}\right) H_{m}^{(2) \prime}\left(k_{1} \rho_{\text {out }}\right)+\frac{k_{2}}{\mu_{2}} H_{m}^{(2) \prime}{ }^{\prime} k_{2} \rho_{\text {out }}\right) H_{m}^{(2)}\left(k_{1} \rho_{\text {out }}\right)\right]
\end{aligned}
$$

Analytical form of the scattering coefficients for a metallic cylinder with a dielectric cover [36]

$$
\begin{gathered}
s_{m}=\frac{\frac{\mu_{2}}{\mu_{1}} k_{1} \alpha J_{m}^{\prime}\left(k_{1} \rho_{\text {out }}\right)-k_{2} \beta J_{m}\left(k_{1} \rho_{\text {out }}\right)}{-\frac{\mu_{2}}{\mu_{1}} k_{1} \alpha H_{m}^{(2)^{\prime}}\left(k_{1} \rho_{\text {out }}\right)+k_{2} \beta H_{m}^{(2)}\left(k_{1} \rho_{\text {out }}\right)} \\
\beta=H_{m}^{(2)}\left(k_{2} \rho_{\text {in }}\right) H_{m}^{(1)^{\prime}}\left(k_{2} \rho_{\text {out }}\right)-H_{m}^{(2)^{\prime}}\left(k_{2} \rho_{\text {out }}\right) H_{m}^{(1)}\left(k_{2} \rho_{\text {in }}\right) \\
\alpha=H_{m}^{(1)}\left(k_{2} \rho_{\text {out }}\right) H_{m}^{(2)}\left(k_{2} \rho_{\text {in }}\right)-H_{m}^{(1)}\left(k_{2} \rho_{\text {in }}\right) H_{m}^{(2)}\left(k_{2} \rho_{\text {out }}\right)
\end{gathered}
$$

\section{References}

1. Fernandes, C.A.; Lima, E.B.; Costa, J.R. Dielectric Lens Antennas. In Handbook of Antenna Technologies; Springer: Singapore, 2016; pp. 1001-1064.

2. Luneburg, R.K. Mathematical Theory of Optics; Brown University Press: Providence, RI, USA, 1944.

3. Maxwell, J.C. The Scientific Papers of James Clerk Maxwelli; Cambridge University Press: Cambridge, UK, 1890; Volume 2.

4. Mosallaei, H.; Rahmat-Samii, Y. Non-uniform Luneburg and two shell lens antennas: Radiation characteristics and design optimization. IEEE Trans. Antennas Propag. 2001, 49, 60-69. [CrossRef]

5. Fuchs, B.; Le Coq, L.; Lafond, O.; Rondineau, S.; Himdi, M. Design optimization of multishell Luneburg lenses. IEEE Trans. Antennas Propag. 2007, 55, 283-289. [CrossRef]

6. Fuchs, B.; Lafond, O.; Rondineau, S.; Himdi, M. Design and characterization of half Maxwell fish-eye lens antennas in millimeter waves. IEEE Trans. Microw. Theory Techn. 2006, 54, 2292-2300. [CrossRef]

7. Ma, H.F.; Chen, X.; Xu, H.S.; Yang, X.M.; Jiang, W.X.; Cui, T.J. Experiments on high-performance beam-scanning antennas made of gradient-index metamaterials. Appl. Phys. Lett. 2009, 95, 094107. [CrossRef]

8. Ma, H.F.; Chen, X.; Yang, X.M.; Jiang, W.X.; Cui, T.J. Design of multibeam scanning antennas with high gains and low sidelobes using gradient-index metamaterials. J. Appl. Phys. 2010, 107, 014902. [CrossRef]

9. Mei, Z.L.; Bai, J.; Niu, T.M.; Cui, T.J. A Half Maxwell Fish-Eye Lens Antenna Based on Gradient-Index Metamaterials. IEEE Trans. Antennas Propag. 2012, 60, 398-401. [CrossRef] 
10. Yang, Z.; Guo, L.; Yao, C.; Zhang, Q.; Xu, Z.; Guo, M.; Wang, Z. Ultrawideband Antipodal Tapered Slot Antenna With Gradient Refractive Index Metamaterial Lens. IEEE Antennas Wirel. Propag. Lett. 2019, 18, 2741-2745. [CrossRef]

11. Papathanasopoulos, A.; Rahmat-Samii, Y.; Garcia, N.; Chisum, J.D. A Novel Collapsible Flat-Layered Metamaterial Gradient-Refractive-Index (GRIN) Lens Antenna. IEEE Trans. Antennas Propag. 2019. [CrossRef]

12. Hu, B.; Wu, T.; Cai, Y.; Zhang, W.; Zhang, B. A Novel Metamaterial-Based Planar Integrated Luneburg Lens Antenna with Wide Bandwidth and High Gain. IEEE Access 2020, 8, 4708-4713. [CrossRef]

13. Pendry, J.B.; Schurig, D.; Smith, D.R. Controlling electromagnetic fields. Science 2006, 312, $1780-1782$. [CrossRef]

14. Greenleaf, A.; Kurylev, Y.; Lassas, M.; Uhlmann, G. Cloaking a sensor via transformation optics. Phys. Rev. E 2011, 83, 016603. [CrossRef] [PubMed]

15. Aghanejad, I.; Abiri, H.; Yahaghi, A. Design of High-Gain Lens Antenna by Gradient-Index Metamaterials Using Transformation Optics. IEEE Trans. Antennas Propag. 2012, 60, 4074-4081. [CrossRef]

16. Wu, Q.; Jiang, Z.H.; Quevedo-Turel, O.; Turpin, J.P.; Tang, W.; Hao, Y.; Werner, D.H. Transformation Optics Inspired Multibeam Lens Antennas for Broadband Directive Radiation. IEEE Trans. Antennas Propag. 2013, 61, 5910-5922. [CrossRef]

17. Moccia, M.; Castaldi, G.; D'Alterio, G.; Feo, M.; Vitiello, R.; Galdi, V. Transformation-Optics-Based Design of a Metamaterial Radome for Extending the Scanning Angle of a Phased-Array Antenna. IEEE J. Multiscale Multiphys. Comput. Tech. 2017, 2, 159-167. [CrossRef]

18. Giloan, M.; Gutt, R. Optical imaging system based on transformation-optics lenses. J. Opt. 2019, $22,015103$. [CrossRef]

19. Eskandari, H.; Quevedo-Teruel, O.; Attari, A.R.; Majedi, M.S. Transformation optics for perfect two-dimensional non-magnetic all-mode waveguide couplers. Opt. Mater. Express 2019, 9, 1320-1332. [CrossRef]

20. Isernia, T.; Palmeri, R.; Morabito, A.F.; Di Donato, L. Inverse scattering and compressive sensing as advanced em design tools. In Proceedings of the IEEE International Symposium on Antennas and Propagation \& USNC/URSI National Radio Science Meeting, San Diego, CA, USA, 9-14 July 2017; pp. 433-434.

21. Palmeri, R.; Bevacqua, M.T.; Morabito, A.F.; Isernia, T. Inverse scattering methods as a tool for the design of GPCs devices. In Proceedings of the IEEE Conference on Antenna Measurements \& Applications, Tsukuba, Japan, 4-6 December 2017.

22. Isernia, T.; Palmeri, R. Inverse scattering as a way to artificial dielectrics based devices. In Proceedings of the International Congress on Artificial Materials for Novel Wave Phenomena, Espoo, Finland, 27-30 August 2018.

23. Palmeri, R.; Isernia, T. Volumetric invisibility cloaks design through spectral coverage optimization. IEEE Access 2019, 7, 30860-30867. [CrossRef]

24. Colton, D.; Kress, R. Inverse Acoustic and Electromagnetic Scattering Theory; Springer: New York, NY, USA, 2012; Volume 93.

25. Bucci, O.M.; Catapano, I.; Crocco, L.; Isernia, T. Synthesis of new variable dielectric profile antennas via inverse scattering techniques: A feasibility study. IEEE Trans. Antennas Propag. 2005, 53, 1287-1297. [CrossRef]

26. Palmeri, R.; Bevacqua, M.T.; Morabito, A.F.; Isernia, T. Design of artificial-material-based antennas using inverse scattering techniques. IEEE Trans. Antennas Propag. 2018, 66, 7076-7090. [CrossRef]

27. Van den Berg, P.M.; Kleinman, R.E. A contrast source inversion method. Inverse Prob. 1997, $13,1607$. [CrossRef]

28. Felbacq, D.; Tayeb, G.; Maystre, D. Scattering by a random set of parallel cylinders. JOSA A 1994, 11, 2526-2538. [CrossRef]

29. Richmond, J. Scattering by a dielectric cylinder of arbitrary cross section shape. IEEE Trans. Antennas Propag. 1965, AP-13, 334-341. [CrossRef]

30. Jones, D.S. Acoustic and Electromagnetic Waves; Oxford University Press: Oxford, UK, 1986.

31. Abramovitz, M.; Stegun, I. Handbook of Mathematical Functions; Applied Mathematics Series 55; Department of Commerce, National Bureau of Standards: Gaithersburg, MD, USA, 1972. 
32. Isernia, T.; Pascazio, V.; Pierri, R. On the local minima in a tomographic imaging technique. IEEE Trans. Geosci. Remote Sens. 2001, 39, 1596-1607. [CrossRef]

33. Palmeri, R.; Bevacqua, M.T.; Morabito, A.F.; Isernia, T. A modified contrast source inversion method for the synthesis of innovative dielectric devices. In Proceedings of the IEEE European Conference on Antennas and Propagation, London, UK, 9-13 April 2018.

34. Isernia, T.; Panariello, G. Optimal focusing of scalar fields subject to arbitrary upper bounds. Electron. Lett. 1998, 34, 162-164. [CrossRef] 
35. Bucci, O.M.; Isernia, T. Electromagnetic inverse scattering: Retrievable information and measurement strategies. Radio Sci. 1997, 32, 2123-2137. [CrossRef]

36. Tang, C. Backscattering from dielectric-coated infinite cylindrical obstacles. J. Appl. Phys. 1957, $28,628-633$. [CrossRef]

(C) 2020 by the authors. Licensee MDPI, Basel, Switzerland. This article is an open access article distributed under the terms and conditions of the Creative Commons Attribution (CC BY) license (http://creativecommons.org/licenses/by/4.0/). 